\title{
Anti-Allergic Effect of Para-Probiotics from Non-Viable Acetic Acid Bacteria in Ovalbumin-Sensitized Mice
}

\author{
Shin Nakamura' ${ }^{1,2}$, Fusako Mitsunaga ${ }^{1,2}$ \\ ${ }^{1}$ Intelligence \& Technology Lab Inc., Kaizu, Japan \\ ${ }^{2}$ Biomedical Institute, NPO Primate Agora, Inuyama, Japan \\ Email: *snakamura@itechlab.co.jp
}

How to cite this paper: Nakamura, S. and Mitsunaga, F. (2018) Anti-Allergic Effect of Para-Probiotics from Non-Viable Acetic Acid Bacteria in Ovalbumin-Sensitized Mice. Food and Nutrition Sciences, 9, 1376-1385. https://doi.org/10.4236/fns.2018.912099

Received: November 27, 2018

Accepted: December 21, 2018

Published: December 24, 2018

Copyright ( $) 2018$ by authors and Scientific Research Publishing Inc. This work is licensed under the Creative Commons Attribution International License (CC BY 4.0).

http://creativecommons.org/licenses/by/4.0/

\section{c) (i) Open Access}

\begin{abstract}
Para-probiotics are ghost probiotics and consist of non-viable microbial cells. Here, a genus of acetic acid bacteria $(\mathrm{AAB})$ was lyophilized to produce a para-probiotic preparation of non-viable (NV) AAB. The NV-AAB orally administered for 49 days ameliorated antigen-induced sneezing and plasma antigen-specific IgE in an ovalbumin-sensitized allergic mouse model. Our findings indicated that NV-AAB, as a para-probiotic functional food, exhibits an anti-allergic effect. The anti-allergic mechanism of NV-AAB was atypical because of the near-absence of an effect on Th1 and Th2 balance in splenocytes. NV-AAB will be a unique para-probiotic food material with an anti-allergic activity.
\end{abstract}

\section{Keywords}

Probiotics, Dead Cell, Functional Food, Treg, IgE

\section{Introduction}

Probiotics are live microbial cells that have a wide range of biological effects mediated through immune regulation and the balance of pro-inflammatory and anti-inflammatory cytokines [1]. Probiotic preparations contain dead cells, but their metabolites can produce a biological response that is often similar to that produced by live cells [2] [3] [4]. This is the crux of the probiotic paradox, where both live and dead cells seem capable of generating a biological response [5] [6] [7]. Para-probiotics are heat-inactivated/killed ghost probiotics that consist of non-viable (NV) microbial cells and/or crude cell extracts with complex cellular components and metabolites. When administered orally or topically in adequate 
amounts, para-probiotics confer a benefit on the animal or human [8] [9]. Para-probiotics modulate anti-inflammatory and beneficial immune responses as effectively as live probiotics [10] [11] [12]. Advantages of para-probiotics compared to probiotics include enhanced safety and longer shelf life.

To prepare para-probiotics, probiotics are inactivated using heat, chemicals (formalin), gamma or ultraviolet rays, sonication, and dehydration by lyophilization [13]. Among these approaches, lyophilization is a simple and effective method to preserve the biological effects in contrast to denaturation that occurs with the application of heat, chemicals, or ultraviolet light to inactivate proteins and/or related components.

Acetic acid bacteria (AAB) are gram-negative bacteria that are widely used to produce acetic acid by the oxidization of ethanol and foods, including nata de coco, tea fungus (Kombucha), and yogurt. These foods reportedly exhibit biological responses that include anti-inflammatory and anti-allergic effects [14] [15]. However, these effects have been demonstrated using the finished food products. The quantity of $\mathrm{AAB}$ in these foods is minute and it is unclear whether the live or dead bacteria are responsible for these beneficial effects.

In this study, we use a lyophilized $\mathrm{AAB}$ product (NV-AAB) as the para-probiotic material to examine its immunomodulating effect. Anti-allergic activity of NV-AAB was evident using an ovalbumin (OVA)-sensitized mouse model. To our knowledge, an anti-allergic effect of NV-AAB has not been described. One prior publication reported the use of AAB lipopolysaccharide [16].

\section{Materials and Methods}

\subsection{Mice}

Four to five female BALB/c mice (7-week-old; 16.4 - 20.2 g B.W., Japan SLC, Inc., Hamamatsu, Japan) per cage were housed under specific pathogen-free conditions (controlled temperature between $18^{\circ} \mathrm{C}$ and $28^{\circ} \mathrm{C}$, and humidity between $30 \%$ and $80 \%$ ). They had ad libitum access to standard laboratory food (FR-2; Funabashi Farm, Inc., Funabashi, Japan). Mice were divided into four groups ( $\mathrm{A} 1$ to $\mathrm{A} 4, \mathrm{n}=10$ per group) as follows: non-sensitized (A1), sensitized without NV-AAB (A2), sensitized with low dose (100 mg/kg orally for 49 days) of NV-AAB (A3), and sensitized with high dose $(500 \mathrm{mg} / \mathrm{kg}$ orally for 49 days) of NV-AAB (A4). All animal examinations were conducted according to the Institutional Animal Care and Committee Guide of Intelligence and Technology Lab.

\subsection{OVA Sensitization of Mice}

Two sensitization protocols were used. First, after 15 days of NV-AAB administration, mice were sensitized three times in the subsequent 14 days by weekly subcutaneous injection with $200 \mu \mathrm{L} /$ head of an OVA emulsion in Alum adjuvant. The emulsion $(100 \mu \mathrm{g} / \mathrm{mL})$ was prepared just prior to use by stirring OVA (A5503, Sigma-Aldrich, St. Louis, MO, USA) in phosphate buffered saline (PBS) followed by the dropwise addition of aluminum hydroxide (Imject 
Alum; Thermo Fisher Scientific, Waltham, MA, USA) at the final concentration of $20 \mathrm{mg} / \mathrm{mL}$ for $30 \mathrm{~min}$. The injections were performed at the lateral tail base of each mouse after they were anesthetized with ketamine hydrochloride and medetomidine hydrochloride. Second, after administration of NV-AAB for 30 days, mice were sensitized seven times in 18 days by intranasal exposure to OVA alone. Prior to the intranasal sensitization, nasal cavities were pre-treated with $1.5 \% \mathrm{~N}$-acetyl-L-cysteine for $5 \mathrm{~min}$ to perturbate the mucus. After washing with PBS, OVA ( $2 \mathrm{mg} / \mathrm{mL}$ in PBS, $10 \mu \mathrm{L}$ for each nasal cavity) was injected in each nostril using a pipet. The non-sensitized control mice were treated as described above, but received PBS instead of OVA.

\subsection{Evaluation of Nasal Symptoms}

Nasal symptoms were evaluated as the number of sneezes provoked by allergen. The mice were individually placed in observation cages and their behavior was monitored by a video camera. Both nasal cavities were challenged with OVA as detailed above. The number of sneezes during $5 \mathrm{~min}$ after intranasal challenge was counted by at least three investigators for each observation session. The average number of sneezes in a session was calculated. In cases where the coefficient variation exceeded $10 \%$, the behavior of the mouse was re-examined by another viewing of the recorded video.

\subsection{Preparation of NV-AAB}

AAB (Gluconacetobacter genus) was obtained from Kewpie Corporation, Tokyo, Japan. The bacteria were cultured at $30^{\circ} \mathrm{C}$ with a medium containing sugar, yeast extract, and ethanol. To obtain the $\mathrm{AAB}$ concentrate, the culture was centrifuged at $10,000 \mathrm{rpm}$ for $4 \mathrm{~min}$ at $5^{\circ} \mathrm{C}$. The bacteria were washed twice by the addition of a 10-fold amount (w/w) of a citrate buffer solution ( $\mathrm{pH} 4)$, followed by sufficient stirring and centrifugation as described above. The final bacterial concentrate was lyophilized to yield a powder comprising NV-AAB for use as the para-probiotic.

\subsection{Preparation of Anti-OVA IgE Standard for ELISA}

In preliminary studies, $\mathrm{BALB} / \mathrm{c}$ female mice were sensitized by OVA as mentioned above to obtain OVA antisera for standard stock. Small aliquots of the stock were stored at $-70^{\circ} \mathrm{C}$ until use. The standard stock was serially diluted two-fold from 1:100 to 1:6400 to obtain preparations with an optical density at $450 \mathrm{~nm}$ from 0.1 to 1.8 measured using the enzyme-linked immunosorbent assay (ELISA) to obtain a standard curve. Arbitrary units were assigned to the standard for stable titration, with the least dilute standard (1:100) as 100 and the most dilute standard (1:6400) as 1.563. The correlation between the dilution and the unit was always $>0.9$.

\subsection{ELISA for Antigen-Specific IgE}

A blood sample was collected from each mouse and centrifuged at $1000 \times \mathrm{g}$ for 
10 min to obtain plasma. The plasma levels of OVA-specific IgE were analyzed using a highly sensitive ELISA utilizing a biotinyltyramide amplification system (PerkinElmer Inc., Waltham, MA, USA). In brief, OVA (A5503, Sigma-Aldrich, $20 \mu \mathrm{g} / \mathrm{mL}$ in PBS) was used to coat wells of Immuno Module Maxisorp 96-well plates (469949; Nalge Nunc International, Rochester, NY, USA). After blocking with bovine serum albumin (BSA, A2153; Sigma-Aldrich), standards and plasma samples diluted 1:300 in 1\% BSA prepared in -PBS were applied. Next, biotin-conjugated rat monoclonal anti-mouse IgE (ab11580; Abcam, Cambridge, UK) prepared at $0.8 \mu \mathrm{g} / \mathrm{mL}$ in $1 \%$ BSA-PBS was reacted followed by successive application of streptavidin-horse radish peroxidase conjugate (SA10001; Invitrogen, Carlsbad, CA, USA), biotynyltyramide (PerkinElmer), and streptavidin-horseradish peroxidase. Color was developed by adding the 3,3',5,5'-tetramethylbenzidine substrate (T8665; Sigma-Aldrich). The enzyme reaction was terminated by sulfuric acid and the plate was read at $450 \mathrm{~nm}$ with subtraction of the reference determined at $630 \mathrm{~nm}$ using an iMark micro plate reader (Bio-Rad Laboratories Inc., Hercules, CA, USA). The plates were washed between each step with $0.05 \%$ Tween-20 in PBS, except when sulfuric acid was added. The antibody titer of sample was calculated by a calibration curve generated by a serially diluted standard.

\subsection{Assay of Splenocytic Cytokines}

Splenic cells were prepared from each mouse in the four groups by the centrifugation of mesh-homogenized spleens in PBS followed by washing with RPMI 1640 medium and suspended in this supplemented with $10 \%$ fetal bovine serum and $5 \mathrm{mg} / \mathrm{mL}$ OVA. The obtained splenic cells were cultured in wells of a 96-well dish $\left(5 \times 10^{5}\right.$ cells/well $)$ at $37^{\circ} \mathrm{C}$ in an atmosphere of under $5 \% \mathrm{CO}_{2}$ and $-95 \%$ air. After $24 \mathrm{~h}$, the culture was centrifuged and the supernatant was collected to assay for the cytokines, interferon-gamma (IFN- $\gamma$, Th1marker), interleukin (IL)-4(Th2 marker), and IL-17 (Th17 marker), using separate ELISA kits (Thermo Fisher Scientific) as described by the manufacturer.

\subsection{Statistics}

Data were expressed as mean \pm standard error (SE). Comparisons were made using a Dunnett's test if the variance was equal and steel test if the variance was unequal.

All the experiments were performed at the author's institute in the period from March 23, 2018 to May 16, 2018.

\section{Results}

Production of IL-4 was markedly increased and production of IFN- $\gamma$ was decreased in splenocytes from mice sensitized with OVA for 49 days (Figure 3(b) and Figure 3(a)), indicating that the Th1-Th2 balance in the sensitized mice reached a Th2 dominant state. 


\subsection{Effect on Sneezing}

Oral administration of NV-AAB significantly reduced the number of sneezes in OVA-sensitized mice $(\mathrm{p}<0.01$, Figure 1$)$. This effect appeared to be dose dependent, with the reduction in sneezing being more pronounced with the 500 $\mathrm{mg} / \mathrm{kg}$ dose than the $100 \mathrm{mg} / \mathrm{kg}$ dose.

\subsection{Effect on OVA-Specific IgE}

NV-AAB administration also clearly lowered the plasma antibody level of OVA-specific IgE in OVA-sensitized mice in a dose dependent manner (Figure 2).

\subsection{Effect on Splenocytic Production of Cytokines}

NV-AAB had little effect on the production of IFN- $\gamma$ and IL-4 in the culture supernatant of OVA-stimulated splenocytes from the OVA-sensitized mice (Figure 3(a) and Figure 3(b)). The IL-17 level in the culture supernatant was almost unchanged (Figure $3(\mathrm{c})$ ). These results indicated that the production of the three cytokines in the splenocytes was unaffected by the administration of $\mathrm{NV}$-ABB to the OVA-sensitized mice.

The ratio of IFN- $\gamma /$ IL-4 (as a marker of Th1/Th2) and IL-17 (as a marker of Th17) were similar in the splenocytes from mice sensitized with OVA, 100 $\mathrm{mg} / \mathrm{kg} \mathrm{NV-ABB}$, and $500 \mathrm{mg} / \mathrm{kg} \mathrm{NV}-\mathrm{ABB}$ (Figure 4). The findings imply that

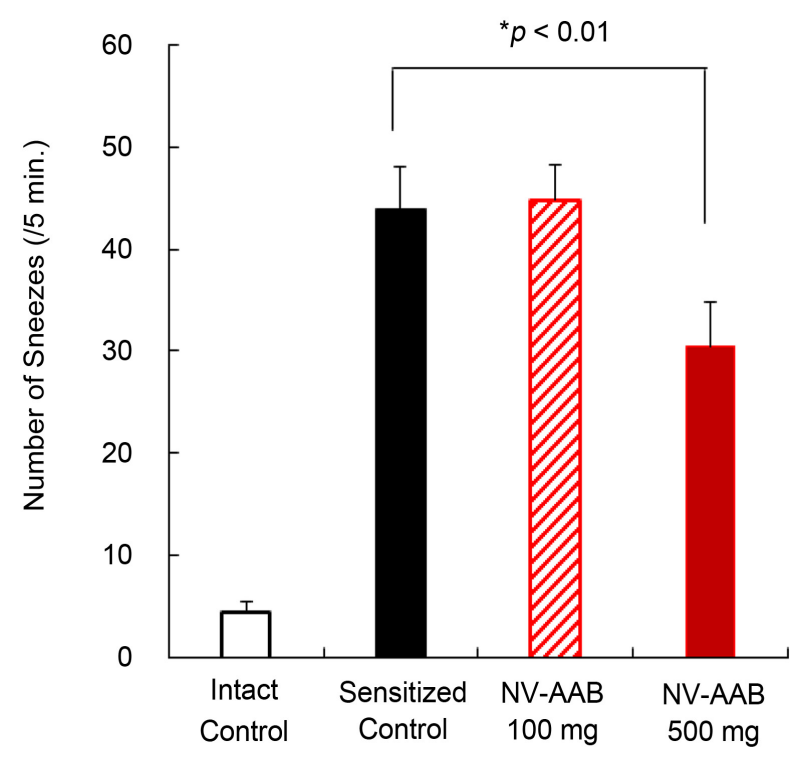

Figure 1. Nasal Symptoms induced by OVA challenge in OVA-sensitized mice. The nasal symptom was evaluated by the number of sneezing provoked by allergen. Open bar: intact, non-OVA-sentized mice; Black filled bar: OVA-sentized mice without NV-AAB; Red hatched bar: OVA-sentized mice administrated with $\mathrm{NV}-\mathrm{AAB}$ at the concentration of 100 $\mathrm{mg} / \mathrm{kg} \mathrm{AV}-\mathrm{AAB}$ and Red filled bar: at the concentration of 500 $\mathrm{mg} / \mathrm{kg}$ AV-AAB. 


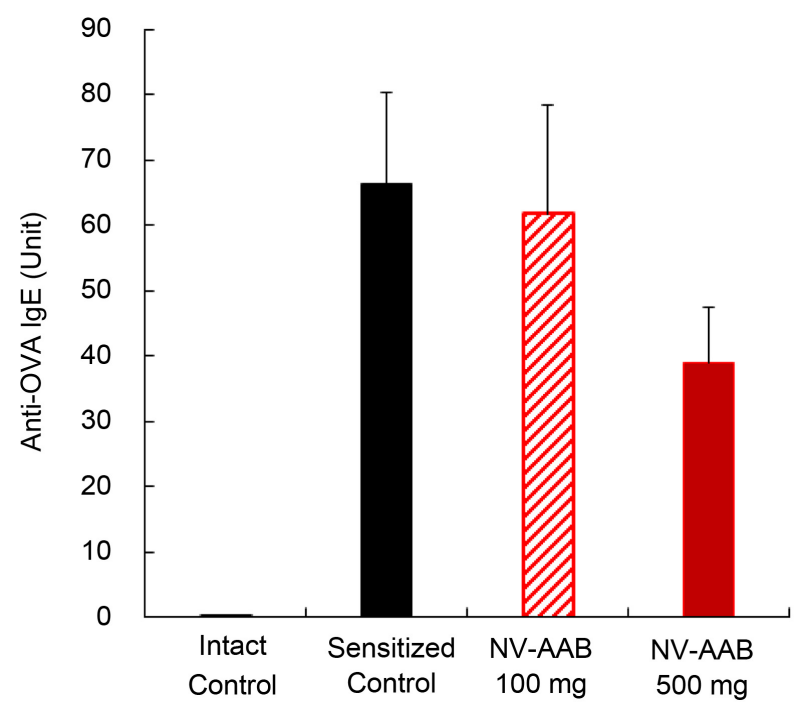

Figure 2. Plasma level of OVA-specific IgE. OVA-specific IgE was assayed by the highly sensitive ELISA described in material and methods using a biotinyltyramide amplification system and the standard for expressing arbitrary unit of OVA-specific IgE. Open bar: intact, non-OVA-sentized mice; Black filled bar: OVA-sentized mice without NV-AAB; Red hatched bar: OVA-sentized mice administrated with NV-AAB at the concentration of $100 \mathrm{mg} / \mathrm{kg} \mathrm{AV}-\mathrm{AAB}$ and Red filled bar: at the concentration of $500 \mathrm{mg} / \mathrm{kg} \mathrm{AV}-\mathrm{AAB}$.

NV-AAB could have a coregulatory effect on the Th1, Th2, and Th17 effector $\mathrm{T}$-cells in the sensitized splenocytes.

\section{Discussion}

Type I allergy is a complex immune-inflammatory disorder that participates in antigen-mediated activation and/or regulation of helper T-cells, effector T-cells (Th1, Th2, and Th17) and regulatory T-cells (Treg). These T-cell events activate B-cells, which produce antigen specific IgE [17]. Antigen-elicited activation of mast cells occurs, followed by the release of several inflammatory factors that cause localized inflammatory symptoms, such as sneezing.

In Japan, pollen allergy has increased in the past few decades and the demand has grown for anti-allergic functional foods and/or related materials, including probiotics. However, probiotics are viable cell preparations. Viability of the cells during the production process can be challenging. By contrast, para-probiotics are non-living cell preparations that can also contain cellular components like lipopolysaccharide. The immune modulating effects of para-probiotics have been described [18] [19] [20]. However, there is little information concerning the anti-allergic action of para-probiotics.

The present study documents the anti-allergic effect of NV-AAB used as a para-probiotic. NV-AAB alleviated antigen-induced sneezing and plasma level of antigen specific IgE in OVA-sensitized mice. The reduced plasma level of 


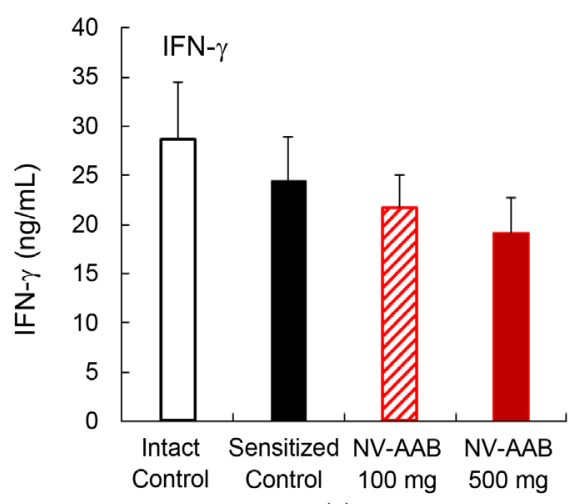

(a)

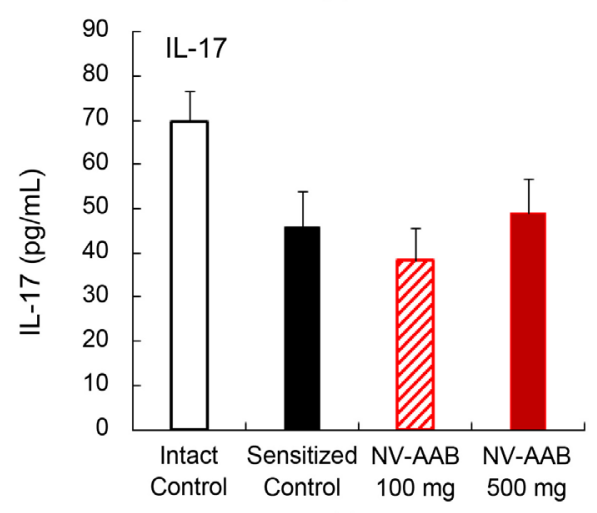

(c)

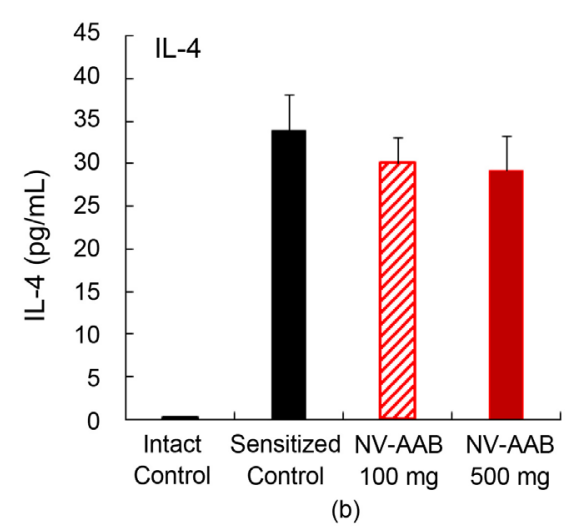

(b)

Figure 3. Cytokine production by OVA-stimulated splenocytes from OVA-sensitized mice without and with oral administration of NV-AAB. Each cytokine, (a) IFN- $\gamma$ (Th1 marker), (b) IL-4 (Th2 marker) and (c) IL-17 (Th17 maker), was assayed using supernatant obtained from culture examination of splenic cells under the conditions mentioned in material and methods. Open bar: intact, non-OVA-sentizedmice; Black filled bar: OVA-sentized mice without NV-AAB; Red hatched bar: OVA-sentized mice administrated with NV-AAB at the concentration of $100 \mathrm{mg} / \mathrm{kg} \mathrm{AV}-\mathrm{AAB}$ and Red filled bar: at the concentration of $500 \mathrm{mg} / \mathrm{kg} \mathrm{AV}-\mathrm{AAB}$.

OVA-specific IgE likely resulted from some inhibitory action of NV-AAB to producing the IgE in B-cells in OVA-sensitized animals. A regulatory effect by effector T-cells on B-cells, with the consequent upregulation of Th1 and/or downregulation of Th2 is also conceivable. However, unexpected results were obtained when we examined the influence of NV-AAB on the production of the IFN- $\gamma$, IL-4, and IL-17 cytokines in splenocytes from OVA-sensitized mice. IFN- $\gamma$, IL-4, and IL-17 are markers of Th1, Th2, and Th17, respectively. Only slight and essentially negligible changes were observed in the level of these three cytokines in the culture supernatant obtained from the splenocytes. These results suggest that regulation by effector T-cells is an implausible explanation for the inhibitory effect of NV-AAB on specific IgE production in B-cells. This contradicts prior descriptions of the upregulation of Th1 and downregulation of Th2 related to anti-allergic functional foods containing probiotic or para-probiotic materials [18] [19] [20].

Treg cells regulate the proliferation and differentiation of the Th1, Th2, 


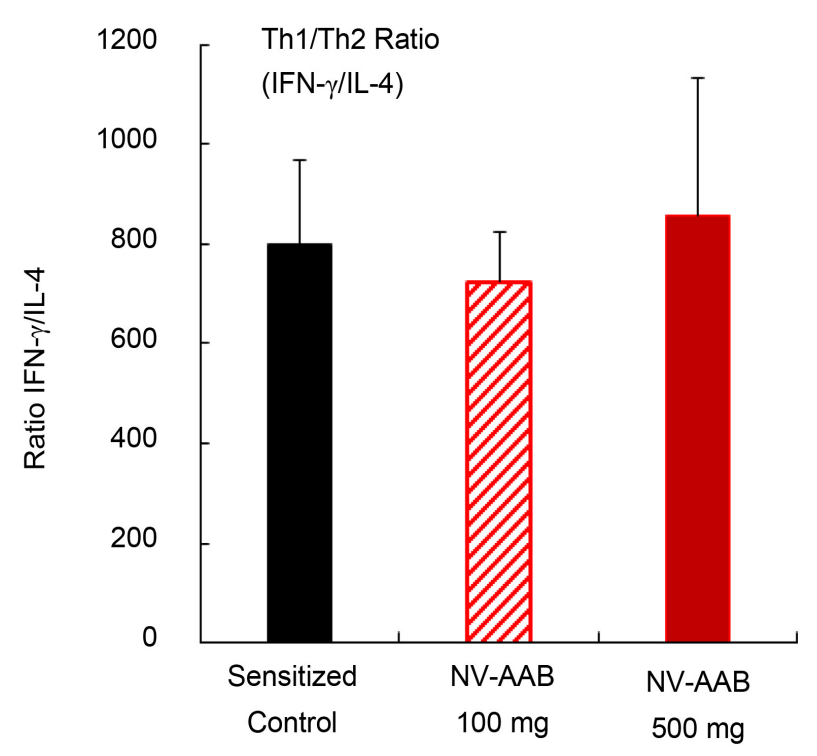

Figure 4. The ratio of Th1 to Th2. The Th1/Th2 ratio was calculated from each producing amount of IFN- $\gamma$ for Th1 and IL-4 for Th2 expressed in Figure 3(a) and Figure 3(b). Black filled bar: OVA-sentized mice without NV-AAB; Red hatched bar: OVA-sentized mice administrated with $\mathrm{NV}-\mathrm{AAB}$ at the concentration of $100 \mathrm{mg} / \mathrm{kg} \mathrm{AV}-\mathrm{AAB}$ and Red filled bar: at the concentration of $500 \mathrm{mg} / \mathrm{kg} \mathrm{AV}-\mathrm{AAB}$.

and Th17 effector T-cells [20]. Treg cells also reportedly inhibit antigen specific IgE production in B-cells [21] [22]. Several bacteria and their products like as short chain fatty acids including acetic acid, promote Treg cells in vivo [23] [24] [25]. Interestingly, in OVA-sensitized mice heat-killed bacteria can induce Treg cells to prevent antigen specific IgE production [26]. Although in this study data concerning the IL-10 Treg cytokine in splenocytes are limited, it is plausible that some common machinery regulates the three effector T-cells, because the same ratio of IFN- $\gamma / \mathrm{IL}-4$ and Th17 was observed in splenocytes. Thus, Treg cells probably participate in the coregulation of Th1, Th2, and Th17 effector T-cells producing IFN- $\gamma$, IL-4, and IL-17, respectively, and in antigen specific IgE production. In further studies, the Treg-associated mechanism of the anti-allergic effect produced by consumption of NV-AAB as a functional food will be determined.

\section{Conclusion}

Oral administration of NV-AAB resulted in anti-allergic activity manifest as the prevention of antigen-induced sneezing and production of plasma antigen specific IgE in OVA-sensitized mice. The results implicate that NV-AAB is an attractive para-probiotic functional food with an anti-allergic effect. This anti-allergic action appears to be unique because of the marginal influence of cytokine production by Th1, Th2, and Th17 effector T-cells in OVA-stimulated splenocytes from the mice administered NV-AAB. 


\section{Conflicts of Interest}

The authors declare no conflicts of interest regarding the publication of this paper.

\section{References}

[1] Isolauri, E., Sütas, Y., Kankaanpää, P., Arvilommi, H. and Salminen, S. (2001) Probiotics: Effects on Immunity. The American Journal of Clinical Nutrition, 73, 444S-450S. https://doi.org/10.1093/ajcn/73.2.444s

[2] Adams, C.A. (2010) The Probiotic Paradox: Live and Dead Cells Are Biological Response Modifiers. Nutrition Research Reviews, 23, 37-46. https://doi.org/10.1017/S0954422410000090

[3] Lahtinen, S.J. (2012) Probiotic Viability: Does It Matter? Microbial Ecology in Health and Disease, 18, 23.

[4] Nighswonger, B.D., Brashears, M.M. and Gilliland, S.E. (1996) Viability of Lactobacillus Acidophilus and Lactobacillus Casei in Fermented Milk Products during Refrigerated Storage. Journal of Dairy Science, 79, 212-219. https://doi.org/10.3168/jds.S0022-0302(96)76353-1

[5] Dotan, I. and Rachmilewitz, D. (2005) Probiotics in Inflammatory Bowel Disease: Possible Mechanisms of Action. Current Opinion in Gastroenterology, 21, 426-430.

[6] Sashihara, T., Sueki, N. and Ikegami, S. (2006) An Analysis of the Effectiveness of Heat-Killed Lactic Acid Bacteria in Alleviating Allergic Diseases. Journal of Dairy Science, 89, 2846-2855. https://doi.org/10.3168/jds.S0022-0302(06)72557-7

[7] Zhang, L., Li, N., Caicedo, R. and Neu, J. (2005) Alive and Dead Lactobacillus Rhamnosus GG Decrease Tumor Necrosis Factor-Alpha-Induced Interleukin-8 Production in Caco-2 Cells. The Journal of Nutrition, 135, 1752-1756. https://doi.org/10.1093/jn/135.7.175

[8] Kimoto-Nira, H., Mizumachi, K., Okamoto, T., Sasaki, K. and Kurisaki, J. (2009) Influence of Long-Term Consumption of a Lactococcus Lactis Strain on the Intestinal Immunity and Iintestinal Flora of the Senescence-Accelerated Mouse. The British Journal of Nutrition, 102, 181-185. https://doi.org/10.1017/S0007114508143574

[9] Moroi, M., Uchi, S., Nakamura, K., Sato, S., Shimizu, N., Fujii, M., et al. (2011) Beneficial Effect of a Diet Containing Heat-Killed Lactobacillus Paracasei K71 on Adult Type Atopic Dermatitis. The Journal of Dermatology, 38, 131-139. https://doi.org/10.1111/j.1346-8138.2010.00939.x

[10] Lopez, M., Li, N., Kataria, J., Russell, M. and Neu, J. (2008) Live and Ultraviolet-Inactivated Lactobacillus Rhamnosus GG Decrease Flagellin-Induced Interleukin-8 Production in Caco-2 Cells. The Journal of Nutrition, 138, 2264-2268. https://doi.org/10.3945/jn.108.093658

[11] Guglielmetti, S., Tamagnini, I., Mora, D., Minuzzo, M., Scarafoni, A., Arioli, S., et al. (2008) Implication of an Outer Surface Lipoprotein in Ahesion of Bifidobacterium Bifidum to Caco-2 Cells. Applied and Environmental Microbiology, 74, 4695-4702. https://doi.org/10.1128/AEM.00124-08

[12] Taverniti, V. and Guglielmetti, S. (2011) The Immunomodulatory Properties of Probiotic Microorganisms beyond Their Viability (Ghost Probiotics: Proposal of Paraprobiotic Concept). Genes \& Nutrition, 6, 261-274.

https://doi.org/10.1007/s12263-011-0218-x

[13] de Almada, C.N., Almada, C.N., Martinez, R.C.R. and Sant'Ana, A.S. (2016) Paraprobiotics: Evidences on Their Ability to Modify Biological Responses, Inactivation 
Methods and Perspectives on Their Application in Foods. Trends in Food Science \& Technology, 58, 96-114. https://doi.org/10.1016/j.tifs.2016.09.011

[14] Lee, M.Y., Ahn, K.S., Kwon, O.K., Kim, M.J., Kim, M.K., Lee, I.Y., et al. (2007) Anti-Inflammatory and Anti-Allergic Effects of Kefir in a Mouse Asthma Model. Immunobiology, 212, 647-654. https://doi.org/10.1016/j.imbio.2007.05.004

[15] Daneshmandi, S., Hajimoradi, M., Soleimani, N. and Sattari, M. (2011) Modulatory Effect of Acetobacter Xylinum Cellulose on Peritoneal Macrophages. Immunopharmacology and Immunotoxicology, 33, 164-168. https://doi.org/10.3109/08923973.2010.491080

[16] Amano, S., Inagawa, H., Nakata, Y., Ohmori, M., Kohchi, C. and Soma, G. (2015) Oral Administration of Lipopolysaccharide of Acetic Acid Bacteria Protects Pollen Allergy in a Murine Model. Anticancer Research, 35, 4509-4514.

[17] Ishii, Y. (2009) Perspective of Prevention and Treatment for Allergic Diseases. Seikagaku, 81, 209-217. (In Japanese)

[18] Dash, G., Raman, R.P., Pani Prasad, K., Makesh, M., Pradeep, M.A. and Sen, S. (2015) Evaluation of Paraprobiotic Applicability of Lactobacillus Plantarum in Improving the Immune Response and Disease Protection in Giant Freshwater Prawn, Macrobrachium rosenbergii (de Man, 1879). Fish \& Shellfish Immunology, 46, 167-174. https://doi.org/10.1016/j.fsi.2014.12.007

[19] Kanauchi, O., Andoh, A., AbuBakar, S. and Yamamoto, N. (2018) Probiotics and Paraprobiotics in Viral Infection: Clinical Application and Effects on the Innate and Acquired Immune Systems. Current Pharmaceutical Design, 24, 710-717. https://doi.org/10.2174/1381612824666180116163411

[20] Palomares, O., Martín-Fontecha, M., Lauener, R., Traidl-Hoffmann, C., Cavkaytar, O., Akdis, M., et al. (2014) Regulatory T Cells and Immune Regulation of Allergic Diseases: Roles of IL-10 and TGF- $\beta$. Genes and Immunity, 15, 511-520. https://doi.org/10.1038/gene.2014.45

[21] Cottrez, F., Hurst, S.D., Coffman, R.L. and Groux, H. (2000) T Regulatory Cells 1 Inhibit a Th2-Specific Response in Vivo. Journal of Immunology, 165, 4848-4853. https://doi.org/10.4049/jimmunol.165.9.4848

[22] Yamamoto, K., Kawamura, I., Tominaga, T., Nomura, T., Ito, J. and Mitsuyama, M. (2006) Listeriolysin O Derived from Listeria Monocytogenes Inhibits the Effector Phase of an Experimental Allergic Rhinitis Induced by Ovalbumin in Mice. Clinical and Experimental Immunology, 144, 475-484. https://doi.org/10.1111/j.1365-2249.2006.03092.x

[23] Atarashi, K., Tanoue, T., Shima, T., Imaoka, A., Kuwahara, T., Momose, Y., et al. (2011) Induction of Colonic Regulatory T Cells by Indigenous Clostridium Species. Science, 331, 337-341. https://doi.org/10.1126/science.1198469

[24] Smith, P.M., Howitt, M.R., Panikov, N., Michaud, M., Gallini, C.A., Bohlooly, Y.M., et al. (2013) The Microbial Metabolites, Short-Chain Fatty Acids, Regulate Colonic Treg Cell Homeostasis. Science, 341, 569-573.

https://doi.org/10.1126/science.1241165

[25] Geuking, M.B., McCoy, K.D. and Macpherson, A.J. (2013) Metabolites from Intestinal Microbes Shape Treg. Cell Research, 23, 1339-1340.

https://doi.org/10.1038/cr.2013.125

[26] Stock, P., Akbari, O., Berry, G., Freeman, G.J., Dekruyff, R.H. and Umetsu, D.T. (2004) Induction of T Helper Type 1-Like Regulatory Cells That Express Foxp3 and Protect against Airway Hyper-Reactivity. Nature Immunology, 5, 1149-1156. https://doi.org/10.1038/ni1122 